\title{
Synchronous Primary Anorectal Melanoma and Sigmoid Adenocarcinoma
}

\author{
Inju Cho, Kyung Jong Kim¹, Sung-Chul Lim \\ Departments of Pathology and ${ }^{1}$ Surgery, Chosun University School of Medicine, Gwangju, Korea
}

A primary anorectal malignant melanoma is a rare tumor. Moreover, cases involving a synchronous anorectal melanoma and colon adenocarcinoma are extremely rare. The authors report a case of a synchronous anorectal melanoma and sigmoid adenocarcinoma in an 84-year-old man. The regions of the anorectal melanoma showed melanocytic nevi in the adjacent mucosa of the anal canal and rectum. A dysplastic nevus was also identified in the anal mucosa. This case demonstrates that an anorectal melanoma can arise from pre-existing anorectal melanocytic lesions.

Keywords: Melanoma; Adenocarcinoma; Anus; Rectum; Sigmoid colon

\section{INTRODUCTION}

An anorectal melanoma is a rare tumor with a poor prognosis. It comprises less than $1 \%$ of all melanomas and about $0.5 \%-2.0 \%$ of all anorectal malignancies. The anorectal region is the third most common site for a malignant melanoma after the skin and the retina [1-3]. Multiple primary colorectal cancers are also rare, with an estimated incidence of about $2.5 \%-5.0 \%$ of all colorectal cancers [3]. A synchronous primary anorectal melanoma and colorectal adenocarcinoma has been very rarely described, with only 6 cases having been reported in the literature [3].

We present a case of a synchronous primary anorectal melanoma and adenocarcinoma of the sigmoid colon. Considerable dispute exists regarding the origin of the cells for the primary anorectal melanoma. The normal mucosa of the lower intestinal tract contains melanocytes, and an increased density of melanocytes in the intestine is frequently found in noncutaneous melanoma patients. This has led to the presumption that the primary anorectal melanoma arises from normal melanocytes in the intes-

Received: February 11, 2016 - Accepted: April 10, 2016

Correspondence to: Sung-Chul Lim, M.D.

Department of Pathology, Chosun University Hospital, 365, Pilmun-daero,

Dong-gu, Gwangju 61453, Korea

Tel: +82-62-230-6343, Fax: +82-62-226-5860

E-mail: sclim@chosun.ac.kr

(C) 2016 The Korean Society of Coloproctology

This is an open-access article distributed under the terms of the Creative Commons Attribution NonCommercial License (http://creativecommons.org/licenses/by-nc/4.0) which permits unrestricted noncommercial use, distribution, and reproduction in any medium, provided the original work is properly cited. tinal epithelium distal to the dentate line and extending proximally into the rectum $[1,4,5]$. This case presents a primary malignant melanoma at the anorectal region associated with melanocytic nevi at the anorectum and an adenocarcinoma of the sigmoid colon.

\section{CASE REPORT}

An 84-year-old Korean man was referred from a local clinic following a positive occult stool blood test. He had no significant medical history except for diabetes mellitus and hyperlipidemia. A physical examination revealed no specific findings, including no skin lesions. Colonoscopy revealed three polypoid lesions in the cecum and transverse colon and an ulcerofungating mass in the sigmoid colon (Fig. 1A). The patient also had a $1.5-\mathrm{cm}$-sized pedunculated polypoid lesion in the proximal anus, a $2-\mathrm{cm}$-sized nodular polypoid mass with superficial ulceration, and another submucosal lesion in the distal rectum just proximal to the anal tumor (Fig. 1B). An endoscopic biopsy showed a moderately differentiated adenocarcinoma in the sigmoid colon (Fig. 1C) and low-grade tubular adenomas in the cecum and transverse colon. Also, among the three lesions of the distal rectum and anus, 2 lesions devoid of any submucosal lesions were biopsied, and those revealed proliferation of epithelioid or spindle cells with brown pigmentation and large nuclei in lamina propria, suggesting a malignant spindle cell tumor (Fig. 1D). However, immunohistochemical (IHC) analysis showed strong positivity for the S-100 protein and HMB45, as well as negativity for cytokeratin, suggesting a malignant melanoma (Fig. 1E). On abdominal computed 

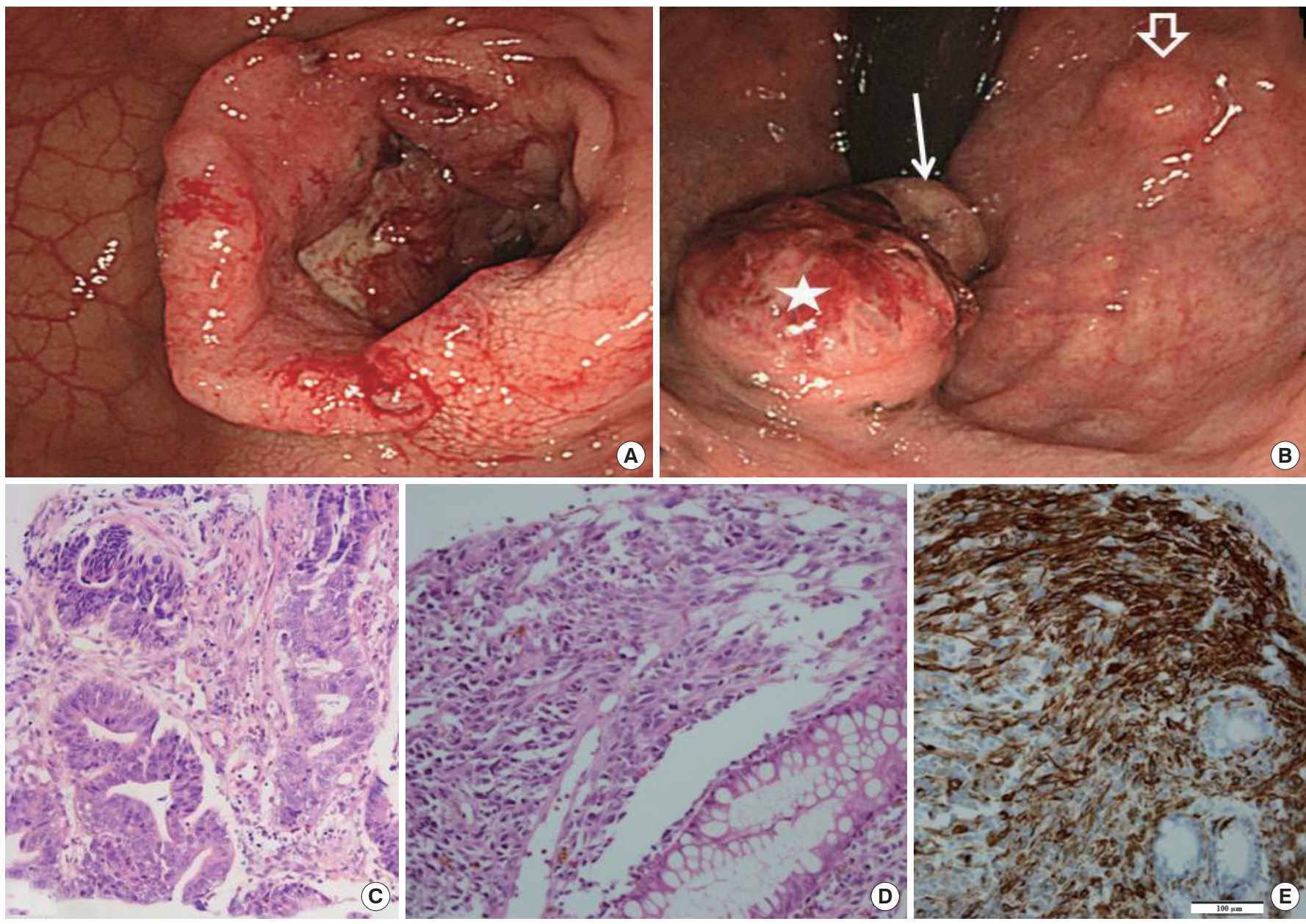

Fig. 1. Endoscopic findings show an ulcerofungating mass in the sigmoid colon (A) and a pedunculated polyp (arrow) in the proximal anus, a nodular mass with superficial ulceration (asterisk), and another submucosal lesion (open arrow) in the distal rectum (B). Endoscopic biopsies show an adenocarcinoma in the colon (C) and a malignant spindle cell proliferation in the rectal mucosa (D) with positivity for HMB-45 immunohistochemical staining (E). The scale bar measures $100 \mu \mathrm{m}$.

tomography (CT), sigmoid colon cancer with multiple lymphnode enlargements was detected. Fluorodeoxyglucose positronemission tomography-CT showed hypermetabolic lesions in the left colon and distal rectum with multiple hypermetabolic lymph nodes in the mesentery, suggesting malignancy with regional lymph-node metastasis.

The patient subsequently underwent a laparoscopic anterior resection and transanal excision (Fig. 2). A pathological examination of a surgical specimen from the anterior resection of the sigmoid colon showed that the tumor was a moderately differentiated adenocarcinoma that had invaded the pericolic adipose tissue. No regional lymph-node metastases or lymphovascular invasion was identified. The transanally excised tumor of the anorectum was almost completely composed of round-to-oval epithelioid cells in the mucosa and the submucosa (Fig. 3), with tumor involvement in the proximal resection margin. The maximal tu-

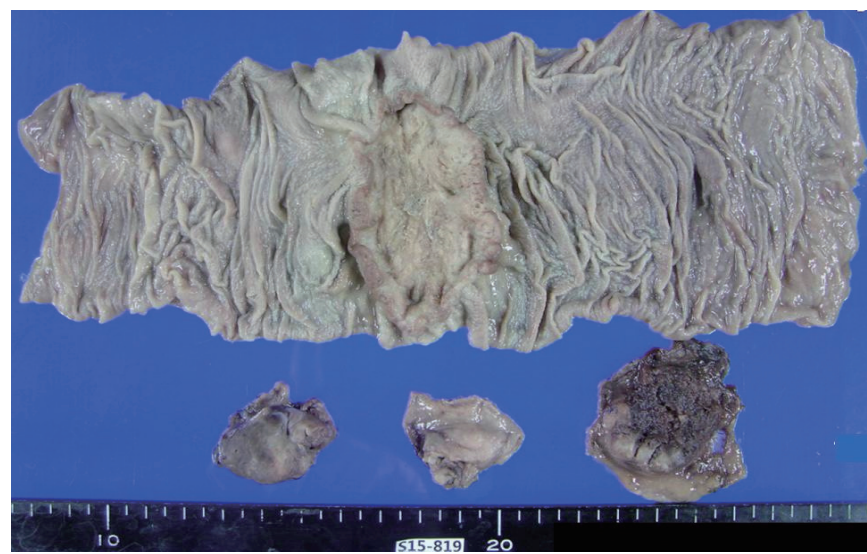

Fig. 2. Gross findings of a laparoscopic anterior resection show an ulcerofungating tumor in the sigmoid colon (upper), and those of the transanal excision show dark-brown-colored ulcerated nodular polypoid lesions (lower). 


\begin{aligned} & Annals of $\begin{array}{l}\text { Synchronous Primary Anorectal Melanoma and Sigmoid Adenocarcinoma } \\ \text { Inju Cho, et al. }\end{array} \\ &$\hline\end{aligned}
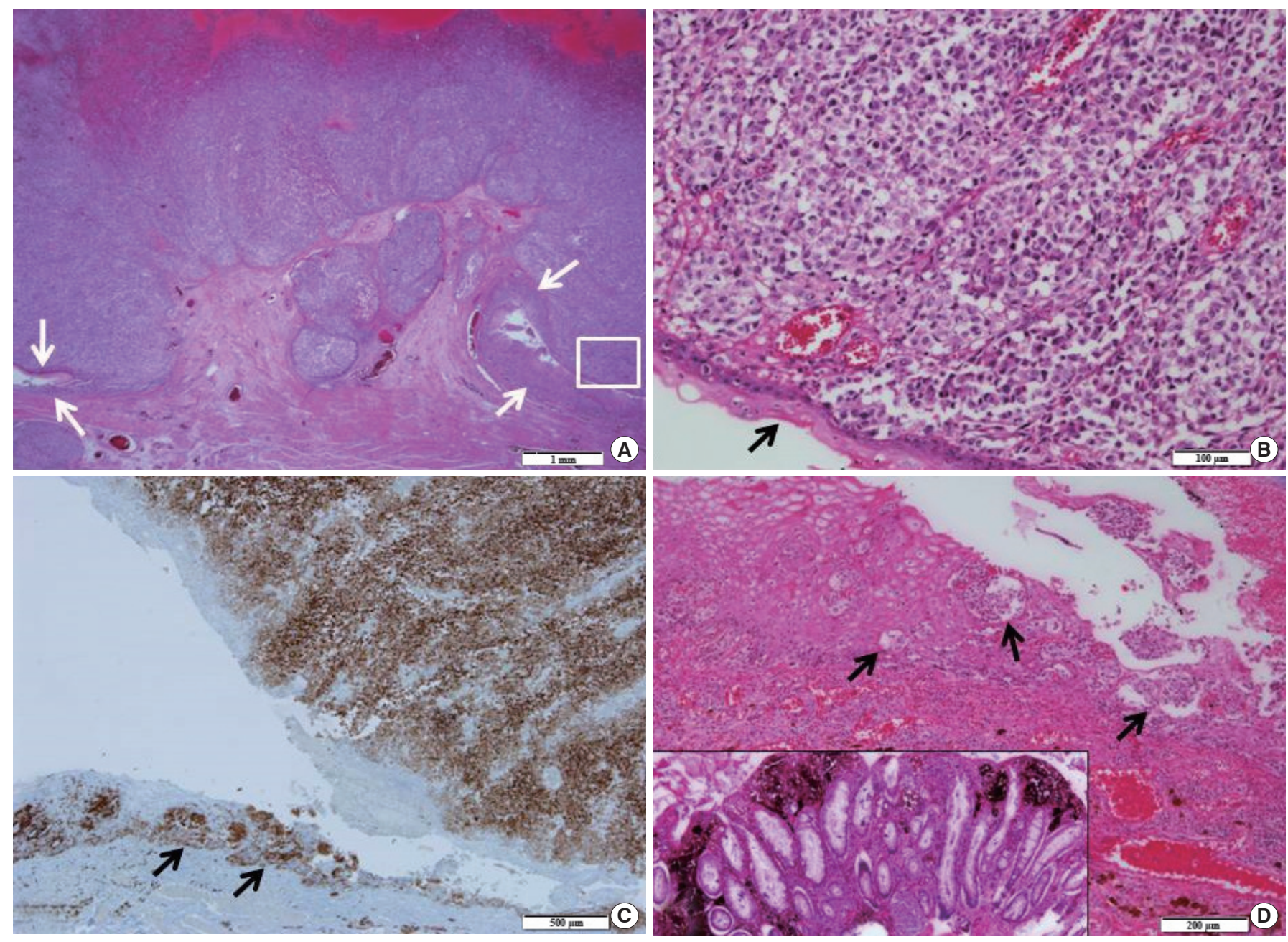

Fig. 3. (A) Microscopic findings show an ulcerofungating tumor involving the anal mucosa and submucosa. Arrows indicate the anal mucosa consisting of a squamous epithelium. The scale bar measures $1 \mathrm{~mm}$. (B) Higher magnification of the boxed area in panel A shows a round atypical cell proliferation with brown pigmentation. The arrow indicates an anal squamous epithelium. The scale bar measures $100 \mu \mathrm{m}$. (C) Immunohistochemical staining for Melan-A demonstrates positive reactions in the anal tumor and the adjacent melanocytic nevus (arrows) in the anal mucosa. The scale bar measures $500 \mu \mathrm{m}$. (D) A melanocytic nevus (arrows) is identified in the squamous epithelium in the anal mucosa. The inset shows a pigmented melanocytic proliferation in the rectal mucosa. The scale bar measures $200 \mu \mathrm{m}$.

mor thickness was $10 \mathrm{~mm}$, and the mitotic count was $40 / 10$ high power field. Lymphovascular invasions were identified. IHC staining of the anorectal tumor showed positivity for Melan A, S-100 protein, and tyrosinase, consistent with a malignant melanoma. Also, an atypical melanocytic proliferation (dysplastic nevus) was identified in the mucosa of the anal canal in a background of anal and rectal melanocytic nevi (Fig. 3).

After surgery, the patient received adjuvant radiotherapy. However, along with local recurrence at the anastomosis site, multiple pulmonary and hepatic metastases were found on his 6-month follow-up CT, and the patient complained of pain despite radiotherapy. He died fourteen months after the diagnosis.

\section{DISCUSSION}

A primary malignant anorectal melanoma is a rare tumor with a dismal prognosis; however, the anorectal region is the third most common area, after the skin and the retina, for such a melanoma. This type of melanoma has a female preponderance, with the highest incidence during the sixth and the seventh decades of life [1-3]. An anorectal melanoma occurs most commonly in the rectum, followed by the anal canal and the anal verge [4]. However, a study conducted by Bello et al. [6] suggested that $65 \%$ of anorectal melanomas were located within the anal canal or the anal verge collectively and that $35 \%$ were located in the distal rectum. In contrast to a cutaneous melanoma, an anorectal melanoma is of- 
ten detected in the late stages of the disease and exhibits different biologic behaviors [3]. Moreover, approximately $20 \%$ of the lesions are amelanotic [4]. Therefore, an anorectal melanoma is associated with poor prognosis [4]. Metastasis is found in up to $70 \%$ of patients at the time of diagnosis; hematogenic metastasis occurs mostly in the liver, lungs, brain, and bones, and regional lymph nodes, including the mesorectal and the inguinal lymph nodes, are often targeted [4].

Yap and Neary [5] classified anorectal melanomas by location. "Anal" referred to a lesion located below the dentate line, "rectal" to a mass located above the dentate line, and "anorectal" to a tumor in or around the dentate line [7]. According to this categorization, Bello et al. [6] studied the clinicopathologic relevance of an anorectal melanoma based on its anatomic location. Lesions proximal to or at the dentate line presented with more advanced stage, and lesions distal to the dentate line more commonly recurred within the lymph nodes. However, all cases of anorectal melanomas showed poor long-term prognosis irrespective of tumor location.

Synchronous colorectal malignancies are also rare, occurring in $3 \%-5 \%$ of patients, with an even lower incidence when Lynch syndrome patients are excluded [3]. Such malignancies are defined as 2 or more distinct tumors diagnosed within 6 months of the initial malignancy that are separated by the normal bowel and not by direct extension or metastasis [3]. A primary anorectal melanoma with a synchronous or concomitant primary colorectal adenocarcinoma has been very rarely described, with only 6 cases having been reported [3]. Lim et al. [3] reviewed those 6 reported cases and found a slight female preponderance, with all patients being older than 50 years. All synchronous colorectal cancers were either in the rectum or the sigmoid. Most patients presented relatively late and with distant metastasis.

Because of its rarity and the late stage at the time of diagnosis, the optimal treatment for an anorectal melanoma is controversial. The aggressive behavior of the tumor has led to the suggestion that an abdominoperineal resection (APR) should be the treatment of choice. Other studies have shown that anal sparing with a wide local excision (WLE) would provide a better quality of life [3, 5]. While no significant differences in survival between the APR and the WLE have been reported, the local recurrence rates were $8 \%$ and $20 \%$ for the APR and the WLE, respectively [5]. The mean survival time has been reported to be 20 months with treatment, and the 5-year disease-free survival rate has been reported to be $6.7 \%-12.0 \%$ with treatment $[2,3]$. Because of the rarity of a synchronous primary anorectal melanoma and colorectal adenocarcinoma, no consensus exists at this time regarding which surgical procedure is preferred. Nilsson and Ragnarsson-Olding [8] found that the resection margin was a significant predictor of long-term survival, with a 5 -year survival rate of $19 \%$ for patients with a clear resection margin (R0 resection), compared to $6 \%$ for those with a positive resection margin ( $\mathrm{R}+$ resection). Notably, patients who had undergone an APR were more likely to have an
$\mathrm{R} 0$ resection (76\%) compared to those who had undergone a WLE (26\%) [8]. Our patient had a WLE for the anorectal melanoma with an R+ resection. The patient had local recurrence at the anastomosis site, and multiple organ metastases developed despite adjuvant radiotherapy. When the fact that patients with a synchronous anorectal melanoma with adenocarcinoma present in a late stage and with multiple metastases is considered, the APR appears to be an appropriate surgical modality for treating a synchronous anorectal melanoma and adenocarcinoma [3].

Despite the multimodality approach, an anorectal melanoma has poor prognosis. The 5 -year disease-specific survival is $<10 \%$, and the mean survival time is 12-18 months [9]. The general prognosis depends on the size and the depth of invasion of the tumor. However, irrespective of location (anal, anorectal, or rectal), the long-term prognosis remains dismal for all patients with an anorectal melanoma $[4,6]$. Whether a patient with a synchronous anorectal melanoma and colorectal adenocarcinoma has a worse prognosis compared to one with solitary colon cancer has not yet been established, primarily due to the rarity of such cases. However, in a study of 339 synchronous colorectal tumors conducted by Passman et al. [10], no difference in survival was noted between patients with solitary colorectal tumors and those with synchronous tumors.

In conclusion, we report the case of a patient with a synchronous anorectal melanoma and sigmoid adenocarcinoma. Such cases are rare, and most patients presents with relatively late-stage disease and with multiple metastases. No consensus currently exists regarding treatment, but, at this time, the APR appears to be the optimal therapy. Early diagnosis is as important as appropriate treatment; thus, a high suspicion of the disease and a thorough evaluation are mandated for a correct diagnosis.

\section{CONFLICT OF INTEREST}

No potential conflict of interest relevant to this article was reported.

\section{ACKNOWLEDGMENTS}

This study was supported by Chosun University Hospital research fund 2016.

\section{REFERENCES}

1. Khan M, Bucher N, Elhassan A, Barbaryan A, Ali AM, Hussain N, et al. Primary anorectal melanoma. Case Rep Oncol 2014;7:16470.

2. Teke Z, Ozogul YB, Aydog G, Dalgic T, Bostanci EB, Akoglu M. Multiple synchronous anorectal malignant melanoma coexisting with adenocarcinoma of the sigmoid colon. Indian J Surg 2013; 75:164-6.

3. Lim A, Grant B, Avramovic J, Ho YH, Wallace C. Synchronous 
primary anorectal melanoma and sigmoid adenocarcinoma: a case report. Int Surg 2015;100:814-7.

4. Heyn J, Placzek M, Ozimek A, Baumgaertner AK, Siebeck M, Volkenandt M. Malignant melanoma of the anal region. Clin Exp Dermatol 2007;32:603-7.

5. Yap LB, Neary P. A comparison of wide local excision with abdominoperineal resection in anorectal melanoma. Melanoma Res 2004;14:147-50.

6. Bello DM, Smyth E, Perez D, Khan S, Temple LK, Ariyan CE, et al. Anal versus rectal melanoma: does site of origin predict outcome? Dis Colon Rectum 2013;56:150-7.

7. Carcoforo P, Raiji MT, Palini GM, Pedriali M, Maestroni U, So- liani G, et al. Primary anorectal melanoma: an update. J Cancer 2012;3:449-53.

8. Nilsson PJ, Ragnarsson-Olding BK. Importance of clear resection margins in anorectal malignant melanoma. Br J Surg 2010;97:98103.

9. Che X, Zhao DB, Wu YK, Wang CF, Cai JQ, Shao YF, et al. Anorectal malignant melanomas: retrospective experience with surgical management. World J Gastroenterol 2011;17:534-9.

10. Passman MA, Pommier RF, Vetto JT. Synchronous colon primaries have the same prognosis as solitary colon cancers. Dis Colon Rectum 1996;39:329-34. 